\title{
Innovation of Energy Technologies of Food Raw Material Dehydration and Extraction
}

\author{
Burdo O.G. ${ }^{1}$, Sirotyuk I.V. ${ }^{1}$, Shcherbich M.V. ${ }^{1}$, Akimov A.V. ${ }^{1}$, Poyan A.S. ${ }^{2}$ \\ ${ }^{1}$ Odessa National Academy of Food Technologies, Odessa, Ukraine \\ ${ }^{2}$ LLC Delta Wilmar Ukraine, Yuzhnyj, Ukraine
}

\begin{abstract}
The researchings of raw material dehydration and extraction are analyzed. The energy problems of product dehydration processes are analyzed. It is shown that recent trends in the development of heat and mass transfer technology are associated with the use of electromagnetic energy generators. The aim of the work is reduction of energy consumption during liquid phase removing from solid plant raw material and reduction of product losses from oil-containing food-industry waste. Achievement of this aim lies with a hypothesis that the use of electromagnetic energy sources in the process of removing moisture from food raw material containing a solid phase will allow to form an additional flow of the liquid phase, in addition to the traditional outlet of the vapor phase. The driving force of such a flow is the effect resulting from the local dissipation of electromagnetic energy in the solid phase volume. A mathematical model of the dehydration process is presented and a set of experimental studies was carried out, which confirmed the validity of the hypothesis. The most significant result of the work is proof of the possibility to organize modes when the juice yield is 4 times higher than the steam yield and, accordingly, to reduce energy consumption for product dehydration. The scientific significance of the obtained results is that a new effect was obtained in the work, which the authors called parodynamic. The practical significance of the work consists of proposing of the technological line for processing oilcontaining waste: coffee sludge, coffee beans husk, reagents (clay and perlite).
\end{abstract}

Keywords: electromagnetic and vacuum technologies, concentration, extraction, dehydration, foodindustry waste.

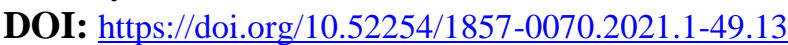

UDC: 620.9-049.34:664.03-027.33: [66.061.3+66.093.48]

Inovarea tehnologiilor energetice pentru deshidratare și extracția materiilor prime alimentare Burdo O.G. ${ }^{1}$, Sirotiuc I.V. ${ }^{1}$, Scerbici M.V. ${ }^{1}$, Akimov A.V. ${ }^{1}$, Poian A.S. ${ }^{2}$

${ }^{1}$ Academia Națională de tehnologii alimentare din Odesa, Odesa, Ucraina

SRL «Delita Vilmar Ucraina», Iujnîi, Ucraina

Rezumat. Se efectuează analiza cercetării în domeniul deshidratării și extracției materiilor prime. Se efectuează compararea dispozitivelor tradiționale pentru deshidratarea materiilor prime. Scopul lucrării este determinat reducerea consumului de energie la eliminarea fazei lichide din materiile prime vegetale solide şi reducerea pierderilor de produse din deșeurile care conțin petrol din producția de alimente. Pentru a atinge acest obiectiv, se propune o idee științifică și tehnică, care se exprimă prin ipoteza că utilizarea surselor de energie electromagnetică în procesul de îndepărtare a umezelii din materiile prime alimentare care conțin o fază solidă va permite, pe lângă ieșirea tradițională a faza de vapori, pentru a forma un flux suplimentar al fazei lichide. Forța motrice a unui astfel de flux este efectul sumar din disiparea locală a energiei electromagnetice în cea mai mare parte a fazei solide. Este prezentat un model matematic al procesului de deshidratare și a fost efectuat un set de studii experimentale, care a confirmat validitatea ipotezei. Cel mai semnificativ rezultat al lucrării este că s-a dovedit că este posibil să se organizeze moduri atunci când randamentul sucului este de 4 ori mai mare decât randamentul aburului și, în consecință, reducerea consumului de energie pentru deshidratarea produsului. Semnificația științifică a rezultatelor obținute este că un nou efect a fost obținut în lucrare, pe care autorii 1-au numit parodinamic. Semnificația practică a lucrării este că se propune o linie tehnologică pentru procesarea deșeurilor care conțin ulei: nămol de cafea, coji de boabe de cafea, reactivi (argilă și perlit).

Cuvinte-cheie: tehnologii electromagnetice și de vid, concentrare, extracție, deshidratare, deșeuri alimentare.

СБурдо О.Г., Сиротюк И.В., Щербич М.В.,

Акимов А.В., Поян А.С. 


\section{Инноватика энергетических технологий дегидратации и экстрагирования пищевого сырья}

Бурдо О.Г. ${ }^{1}$, Сиротюк И.В. ${ }^{1}$, Щербич М.В. ${ }^{1}$, Акимов А.В. ${ }^{1}$, Поян А.С. ${ }^{2}$

${ }^{1}$ Одесская национальная академия пищевых технологий, Одесса, Украина

${ }^{2}$ Общество с ограниченной ответственностью «Дельта Вилмар Украина», Южный, Украина

Аннотация. Проведен анализ исследований в направлении обезвоживания и экстрагирования сырья. Выполнено сравнение традиционных аппаратов для обезвоживания сырья. Сформулированы проблемы пищевых технологий производства концентратов. Анализируются энергетические проблемы процессов обезвоживания продуктов. Определены причины потерь целевых компонентов с отходами производства. Показано, что в последнее время тенденции развития тепломассобменной техники связаны с использованием электромагнитных генераторов энергии. Определена цель работы - снижение затрат энергии при удалении жидкой фазы из твердого растительного сырья и сокращение потерь продукта из маслосодержащих отходов пищевых производств. Для достижения поставленной цели предложена научно-техническая идея, которая выражена гипотезой, что применение электромагнитных источников энергии в процессе удаления влаги из пищевого сырья, содержащего твердую фазу, позволит, кроме традиционного выхода паровой фазы, сформировать дополнительный поток жидкой фазы. Движущей силой такого потока является эффект, возникающий в результате локальной диссипации электромагнитной энергии в объеме твердой фазы. Представлена математическая модель процесса дегидратации и проведен комплекс экспериментальных исследований, который подтвердил справедливость гипотезы. Наиболее существенным результатом работы является то, что доказана возможность организовать режимы, когда выход сока в 4 раза выше, чем выход пара и соответственно снизить расход энергии на обезвоживание продукта. Научная значимость полученных результатов в том, что в работе получен новый эффект, который авторы назвали пародинамический. Практическая значимость работы в том, что предложена технологическая линия переработки маслосодержащих отходов: кофейного шлама, шелухи кофейных зерен, реагентов (глины и перлита). Линия включает микроволновые аппараты для дегидратации и экстрактор. Представлены термограммы и кинетические зависимости исследуемых процессов и объектов. Даны результаты проверки качественных характеристик полученных образцов.

Ключевые слова: электромагнитные и вакуумные технологии, концентрирование, экстрагирование, обезвоживание, отходы пищевых производств.

\section{ВВЕДЕНИЕ}

Современный этап развития пищевой индустрии характеризуется динамичным ростом интереса к пищевым концентратам, ужесточением регламентаций по экологической безопасности производства $[1,2]$, повышением эффективности использования ресурсов (энергетических и сырьевых [3-6]). Комплекс таких задач, иногда противоречивых, предопределяет необходимость поиска новых, инновационных путей интегрированного решения проблем энергетической эффективности, экологии и переработки отходов производства. Самостоятельный интерес представляют отходы пищевых производств. Часто они содержат значительное количество функциональных компонентов, коммерческая привлекательность которых даже выше, чем у готового продукта. Однако традиционные технологии не в состоянии их извлекать, поскольку целевые компоненты сосредоточены в микро- и нанокапиллярах и «вяло» взаимодействуют с экстрагентами. В работе выдвигается концепция, что использование инновационных генераторов энергетического воздействия на пищевое сырье способно решать в комплексе задачи энергетики, экологии и резервных источников пищи.

\section{АНАЛИЗ ЛИТЕРАТУРНЫХ ИСТОЧНИКОВ, ФОРМУЛИРОВКА ПРОБЛЕМЫ И ГИПОТЕЗ}

В технике концентрирования растворов используются 3 принципа удаления влаги: механический, тепловой и диффузионный. Механические аппараты характеризуются минимальными энергетическими затратами на процесс так как протекают без фазового перехода. К недостаткам фильтрующих мембран относятся: малая производительность, ограниченное значение конечной концентрации и проблемы производства мембран. Тепловые (выпаривание) отличаются относительной простотой конструкции и эксплуатации, большой производительностью. К недостаткам выпарных аппаратов относятся: значительные энергетические затраты (необходимые для перевода влаги в пар), ограниченное значение конечной концентрации и опасность порчи термолабильных компонентов сырья. К 
диффузионным относится процесс криоконцентрирования, который характеризуется низкими затратами энергии и полным сохранением функциональных свойств компонентов. Однако оборудование требует высоких капитальных затрат, имеет ограниченное значение конечной концентрации. Кроме того, в аппаратах блочного вымораживания пока не решены задачи реализации тоннажного производства.

Для концентрирования пищевых растворов чаще всего применяются выпарные аппараты. Однако с повышением концентрации раствора растет его вязкость и уменьшается интенсивность теплопередачи (Burdo O.G. et al., Surface Engineering and Applied Electrochemistry, 2018). При наличии твердой фазы для обезвоживания сырья применяют технологии сушки, теория которой отличается значительной сложностью среди всех массообменных процессов. Кроме того, сушка характеризуется и высокими затратами энергии. Например, энергетический КПД самой простой выпарки составляет $85 \%$, а лучших сушильных технологий - в разы меньше. Причина в известном противоречии сушильных технологий. Задача повышения интенсивности процессов переноса связана с ростом скорости потока теплоносителя. А это требует повышения расхода теплоносителя и, как следствие, роста потерь энергии с отработанным теплоносителем (Бурдо О.Г. и др., Электронная обработка материалов, 2020).

В последнее время наметился стабильный рост интереса к применению электромагнитных источников энергии для интенсификации процесса обезвоживания. Так, в 2016 г. в Газиантепском университете (Турция) были проведены эксперименты по изучению влияния микроволнового (МB) выпарного аппарата (BA) на цвет и содержание фенольных компонентов в черничном соке. Для проведения экспериментов было взято 75 мл сока с начальной концентрацией $10^{\circ}$ Brix. Исследования проводились при двух мощностях МВ поля: 200 и 250 Вт. Итоговая концентрация составляла $65^{\circ}$ Brix. Температура выпаривания была в пределах $90^{\circ} \mathrm{C}$ [7].

В 2017 г. ученые Мунзурского университета (Турция) применили СВЧ нагрев при концентрировании яблочного сока. Исследования проводились при различных уровнях подводимой мощности МВ поля (334668 Вт) и давления (500-800 мБар). В МВ ВА помещалась емкость с 450 мл яблочного сока с концентрацией $11^{\circ}$ Brix. B результате выпаривания был получен концентрат с содержанием сухих веществ $66,5^{\circ}$ Brix. В ходе исследований учеными был определен оптимальный режим параметров процесса выпаривания: 668 Вт и 500мБар. Температура при таком сочетании значений параметров составляла $82^{\circ} \mathrm{C}$ [8].

В 2018 г. там же на примере апельсинового сока были продемонстрированы перспективные возможности $\mathrm{MB}$ поля в области концентрирования растворов. Комбинированное применение МВ поля (334 Вт) и разряжения (750 мБар) дало возможность повысить концентрацию сока до $42^{\circ}$ Brix при температуре $65^{\circ} \mathrm{C}$ [9].

В 2019 г. в университете Акдениз (Турция) проводились исследования процесса выпаривания в условиях электромагнитного поля. В качестве пищевых растворов выступали соки винограда (начальная концентрация $\left.\mathrm{X}_{\mathrm{H}}=15,93^{\circ} \mathrm{Brix}\right)$, граната $\left(\mathrm{X}_{\mathrm{H}}=\right.$ $13,91^{\circ}$ Brix) и черной моркови (ХН $=$ $11,23^{\circ}$ Brix).

Начальный объем каждого образца составлял 400 мл. Опыты проводились для двух уровней мощности МВ поля: 180 и 300 Вт. Конечная концентрация каждого образца была порядка $65^{\circ}$ Brix. Данные о температурах растворов в процессе выпаривания в статье не предоставлены [10].

В 2019 г. группа ученых Университета сельского хозяйства (Пакистан), Университета Объединенных Арабских Эмиратов (ОАЭ), Государственного колледжа (Пакистан) и Университета COMSATS в Исламабаде (Пакистан) провели эксперименты по выпариванию сока сахарного тростника в МВ поле. В МВ камеру помещалось 200 мл образца сока и выпаривалось до концентрации $75^{\circ}$ Brix. Процесс обеспечивался при различных уровнях мощности СВЧ излучения $(30,50,80$ и 100 Вт). Данные о температурах образцов не предоставлено [11].

В 2020 г. ученые Малайзийского университета Путра (Малайзия), Университета сельского хозяйства (Пакистан) и Университета Маэджо (Таиланд) исследовали процесс выпаривания кокосового молока с использованием МВ технологий. В камеру МВ ВА помещалось 100 мл кокосового 
молока с концентрацией $12,4^{\circ} \mathrm{Brix}$. Процесс длился по достижению концентрации сока в $65^{\circ}$ Brix. Температура продукта в ходе выпаривания держалась на отметке $103^{\circ} \mathrm{C}$ [12].

В 2020 г. в Исфаханском технологическом университете (Иран) был получен опыт применения МВ ВВА для концентрирования сока барбариса. Исследования проводились при атмосферном давлении и при разряжении в 30 кПа. Конечная концентрация готового продукта составляла $60^{\circ}$ Brix. Значения температур продукта не предоставлены [13].

В 2015 г. в Горганском университете сельскохозяйственных наук (Иран) было изучено изменение цветовых параметров и мутности кизилового сока после концентрирования в MB ВА. Начальные объем и концентрация кизилового сока составляли 400 мл и $7^{\circ}$ Brix соответственно. Опыты проводились при различных уровнях разряжения (12, 38,5 и 100 кПа). Мощность MB поля составляла 300 Вт. По окончании экспериментов был получен сок концентрацией $42^{\circ}$ Brix. Температура при различных значениях разряжения составляла 60,82 и $102^{\circ} \mathrm{C}$ соответственно [14].

В 2011 г. в Тегеранском университете (Иран) были исследованы возможности МВ технологий при выпаривании гранатового сока. За основу было взято 600 мл гранатового сока с начальной концентрацией $14,2^{\circ}$ Brix. Выпаривание проводилось при трех уровнях разряжения (12, 38,5 и 100 (атмосферное) кПа). Мощность электромагнитного излучателя составляла 300 Вт (Обусловлена тем, что при повышении мощности наблюдалось активное пенообразование и пригорание продукта, существенно ухудшающее процесс выпаривания). Конечная концентрация полученного продукта составляла $40^{\circ}$ Brix. Температуры продукта для трех уровнях разряжения в течение процесса колебались в пределах 60$65,75-80$ и $95-105^{\circ} \mathrm{C}$ из-за повышения концентрации продукта и, соответственно, повышения температуры кипения раствора [15].

В 2011 г. там же проводились эксперименты по выпариванию сока черной шелковицы в условиях МВ поля и вакуума. В качестве исходного продукта было взято 600 мл сока черной шелковицы с начальным содержанием сухих веществ $16^{\circ}$ Brix. В ходе эксперимента обеспечивалось три степени разряжения (7,3, 38,5 и 100 (атмосферное) кПа). Во избежание пенообразования и пригорания продукта оптимальным уровнем подводимой мощности МВ поля было принято 300 Вт. В результате был получен концентрат сока с общим содержанием сухих веществ $42^{\circ}$ Brix. Данные о температурах отсутствуют [16].

Положительные результаты использования ИК и МВ генераторов при экстрагировании, выпаривании и сушке пищевого сырья представлены в работах авторов [17-19].

В 2020 г. силами научных сотрудников Технологического университета в Хэфэе, Пекинского университета технологий и бизнеса и Пекинского университета химических технологий (Китай) было изучено влияние $\mathrm{MB}$ технологий на процесс экстрагирования зеленого чая. Процесс протекал при температуре до $70^{\circ} \mathrm{C}$, что является весьма благоприятным для зеленого чая.

Полученный экстракт обладал высоким содержанием кофеина (до 87\%) и прочих полезных компонентов [17].

В 2018 г. учеными университета Улудаг в Бурсе (Турция) были проведены исследования процесса сушки куркумы посредством применения MB (диапазон мощности магнетрона 90-350 Вт), ИК (диапазон рабочих температур $60-80^{\circ} \mathrm{C}$ ) и низкотемпературных (HT) технологий (температура процесса составляла $-50^{\circ} \mathrm{C}$ при давлении 52 Па).

В результате проведения сравнительной характеристики было установлено, что применение MB сушки является наиболее эффективным в силу наименьших затрат времени: 65 минут при мощности 350 Вт в сравнении с ИК (120 минут при температуре $\left.120^{\circ} \mathrm{C}\right)$ и НТ технологиями (600 минут) [18].

В 2016 г. в Анкарском университете (Турция) была организована сушка банана и киви путем комбинирования ИК и МВ источников энергии, мощность излучения которых составляла 600 Вт и $360-420$ Вт соответственно. Эксперименты проводились в сравнении с конвективной (при $70^{\circ} \mathrm{C}$ на протяжении 480 минут) и МВ (с подводимой мощностью в 180-360 Вт для банана и 350-490 Вт для киви) технологиями сушки [19].

Выполненный выше анализ показывает, что механический отжим является наименее энергозатратным процессом удаления жидкости из твердого сырья. Но отжим сопровождается полной деструкцией сырья и 
ощутимыми потерями целевых компонентов с отходами. Сушка сохраняет структуру сырья, но требует существенных затрат энергии. В работе поставлена задача найти эволюционные пути развития техники обезвоживания, перейти от конвективных принципов к инновационным, снять с воздуха задачу теплоносителя и оставить только задачу диффузионной среды.

Решение задачи основано на научнотехнической идее: «Удаление жидкой фазы из твердого сырья возможно за счет создания внутри объема сырья избыточного давления, которое формируется при частичном вскипании в глубине капилляров, пор, межклеточного пространства». Практическое решения задачи основано на использовании соответствующих источников энергии, которые в состоянии инициировать гибридный процесс, который обеспечит точечное парообразование и скачкообразный рост давления. В результате произойдет механический выход потока жидкости из объема твердой фазы. В качестве инновационных рассматриваются источников энергии генераторы микроволнового диапазона, которые реализуют гибридный процесс и пародинамический эффект.

\section{ИННОВАЦИОННАЯ \\ ЭНЕРГОТЕХНОЛОГИЯ УДАЛЕНИЯ ЖИДКОЙ ФАЗЫ ИЗ ТВЕРДОГО СЫРЬЯ}

Для решения задачи сформулирована гипотеза: «Применение электромагнитных источников энергии в процессе удаления влаги из пищевого сырья, содержащего твердую фазу, позволит, кроме традиционного выхода паровой фазы, сформировать дополнительно своеобразный поток жидкой фазы, движущей силой которого является пародинамический эффект, возникающий в результате локальной диссипации электромагнитной энергии в объеме твердой фазы». Фактически, такой поток жидкой фазы для пищевого сырья будет представлять его сок. Рассмотрим физическую схему, которая иллюстрирует гипотезу (рис. 1).
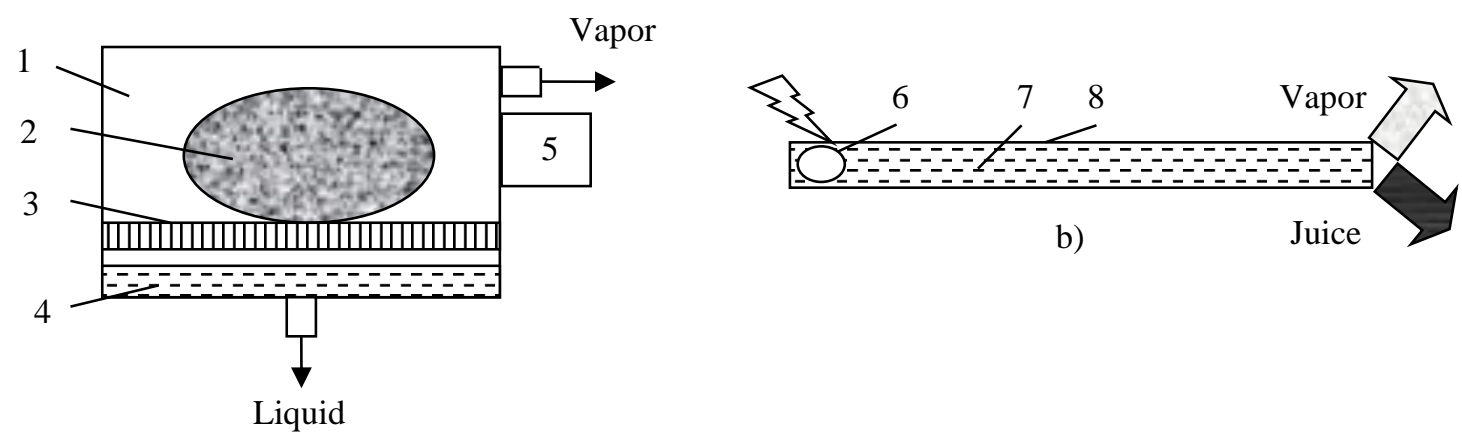

a)

а) физическая модель установки; b) модель «пародинамического эффекта»

Рис. 1. Схема процесса дегидратации. ${ }^{1}$

Анализируемый процесс удаления жидкой фазы, по сути, не является ни классической сушкой (он не диффузионный), ни выпариванием [20]. Точнее его называть процессом дегидратации. Использование электромагнитных источников энергии приводит задачу и к другим граничным (ГУ) условиям. При ГУ 2 рода проходит диссипация электромагнитной энергии (рис.1б) в теплоту. В результате внутри капилляра 8, заполненного жидкостью 7 , формируется паровой пузырек 6. В глубине капилляра растет давление, которое инициирует пародинамический эффект жидкость выбрасывается из открытого торца капилляра в среду. Таким образом, на выходе капилляра два потока: пара и жидкости. Впервые такой эффект заявлен в работе авторов (Burdo O.G., Journal of Engineering Physics and Thermophysics, 2005), дальнейшее развитие теории бародиффузии проведено в (Бурдо О.Г. Пищевые наноэнерготехнологии, 2013; Бурдо О.Г. Эволюиия сушильных установок, 2010), а доказательство его существования видеосъемкой - в (Бурдо О.Г. и др., Проблемы региональной энергетики, 2018). Установлены интервалы формирования пузыря и времени полного осушения канала.

Представляется, что запись нестационарной математической модели 
такого процесса дегидратации при ГУ 2 рода корректна при таких допущениях.
1. Полярные
молекулы
равномерно распределены по объему продукта. Поэтому поток электромагнитной энергии, поступающей в камеру, расходуется на повышение внутренней энергии продукта и рассматривается как равномерно распределенные по объему внутренние источники энергии.

2. Все физические характеристики в процессе обезвоживания являются непрерывными функциями координат и времени.

3. Моделирование процессов в капиллярах и в межклеточном пространстве проводится в цилиндрических координатах.

4. Действием гравитационных и инерционных сил в объеме и теплообменом с окружающей средой пренебрегается.

$\mathrm{B}$ такой постановке при начальных условиях $(\tau=0): t_{1}=t_{i} ; V_{1}=V_{i} ;$ нестационарное поле температур для процесса нагрева жидкости записывается в форме:

$\frac{\partial t_{1}}{\partial \tau}=a_{1}\left(\frac{\partial^{2} t_{1}}{\partial r^{2}}+\frac{1}{r} \frac{\partial t_{1}}{\partial r}+\frac{1}{r^{2}} \frac{\partial^{2} t_{1}}{\partial \phi^{2}}+\frac{\partial^{2} t_{1}}{\partial z^{2}}\right)+\frac{N \eta}{V_{1} c_{1 V} \rho_{1}},(1)$

Формирование пузырька пара характеризуется постоянством температуры фазового перехода $\left(t_{p}=\right.$ const), а подведенная энергия $(N \eta \tau)$ расходуется на повышение внутренней энергии:

$$
N \eta=W_{V} t_{p}\left(c_{L}-c_{V}\right)=W_{V} r
$$

В результате скачкообразно повышается давление:

$$
P(\tau)=P a+\Delta P
$$

В соотношениях $(1-3): c-$ удельная теплоемкость; $\lambda-$ коэффициент теплопроводности; $a-$ коэффициент температуропроводности; $N-$ мощность электромагнитного генератора; $\eta$ - КПД магнетрона; $\tau$ - время работы; $W$ - расход пара; $r$-удельная теплота фазового перехода; $L$ - жидкость; $V$ - пар; $z, r, \varphi$ - координаты.

Уровень роста давления $(\Delta P)$ определяется основным уравнением гидравлики дополненным капиллярным потенциалом канала [13]:

$$
\Delta P=\frac{\rho w^{2}}{2}\left[\frac{\lambda l}{d}+\Sigma \zeta\right]+\rho g l+\frac{\sigma}{d}
$$

где: $l$ - длина каналов, $d$ - их диаметр, $\lambda-$ коэффициент трения, $\zeta$ - сумма местных гидравлических сопротивлений; $\sigma$ коэффициент поверхностного натяжения.

В результате объем жидкости в капилляре уменьшается и его текущее значение определяется уравнением материального баланса:

$$
V_{1}(\tau)=V_{H}-\left(V_{V}-V_{L}\right)(\tau)
$$

Причем, уменьшение объема может быть следствием выброса объема пара $\left(V_{V}\right)$ и части жидкости $\left(V_{L}\right)$ из капилляра.

Сформулированная гипотеза подтверждена экспериментально. В соответствии с рисунком 1 собран стенд, который включал вакуумную резонаторную камеру, микроволновой генератор энергии $(E G)$, холодильную установку ( $C M)$, систему вакуумирования $(V P)$, датчики температуры пара (S4) и жидкости ( $S 3$ ) (рис. 2).

Продукт устанавливался на тефлоновую подставку (TP), вторичный пар конденсировался в холодильникедистилляторе $(C)$. Сок и конденсат собирались в отдельные емкости, установленные на весах TBE-0,21-0,01. Текущие значения веса (датчики $S 1$ и $S 2$ ) и температур поступали на систему сбора информации (MCC), собранную на базе планшета CHUWI CW1506. Потребляемая электрическая энергия измерялась с помощью портативного электронного счетчика, a замеры температуры продукта - пирометром Benetech GM320, измерение давления c помощью образцовых вакууметров ВО ГОСТ 6521-60. Периодически проводились измерения концентрации сока и гидролата электронным рефрактометром HI 96801. Опыты проводились на высоковлажном сырье - бананы и арбуз. Диапазон изменения давления в камере $10 \ldots 110$ кПа, температур $40 \ldots 96^{\circ} \mathrm{C}$. 


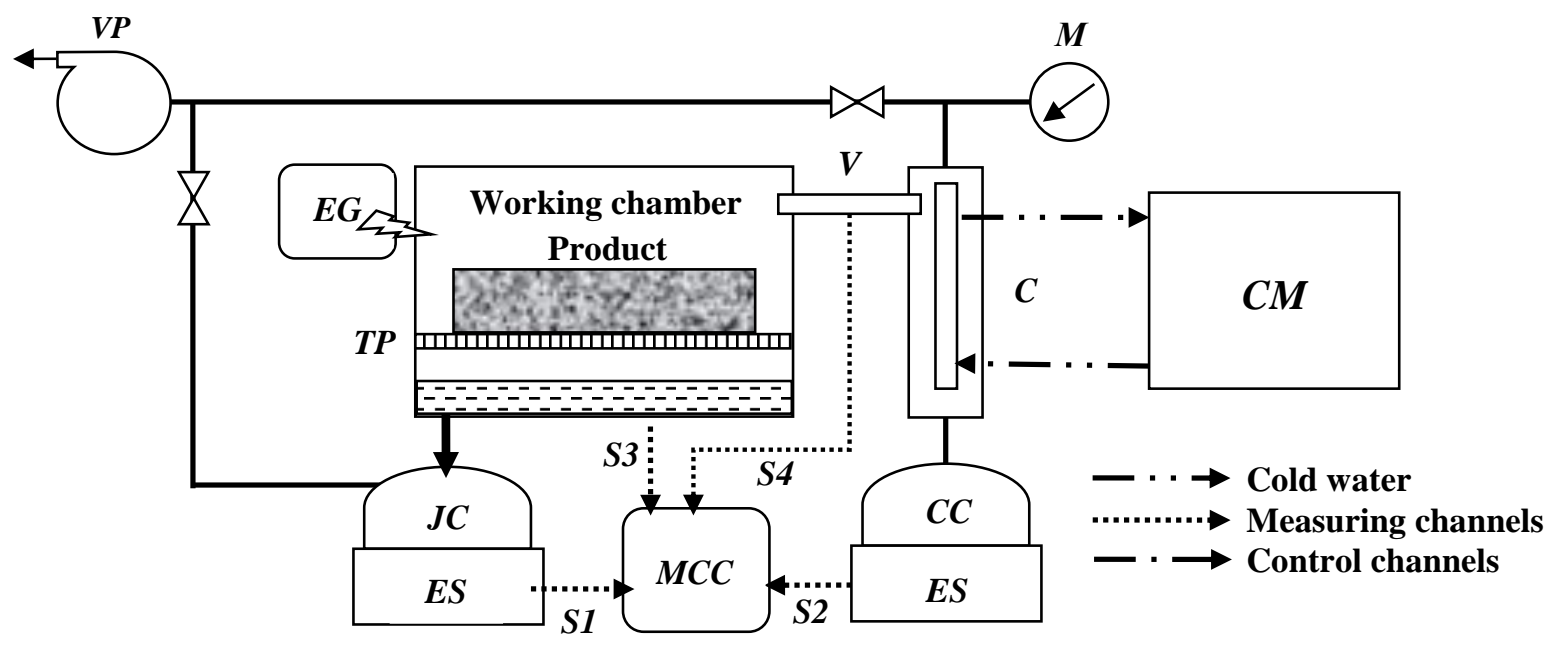

Рис. 2. Схема экспериментального стенда. ${ }^{2}$

В результате пародинамического эффекта сок стекал на дно камеры, откуда отводился в емкость благодаря разности давлений в камере с продуктом и в емкости для сбора сока (рис. 3).

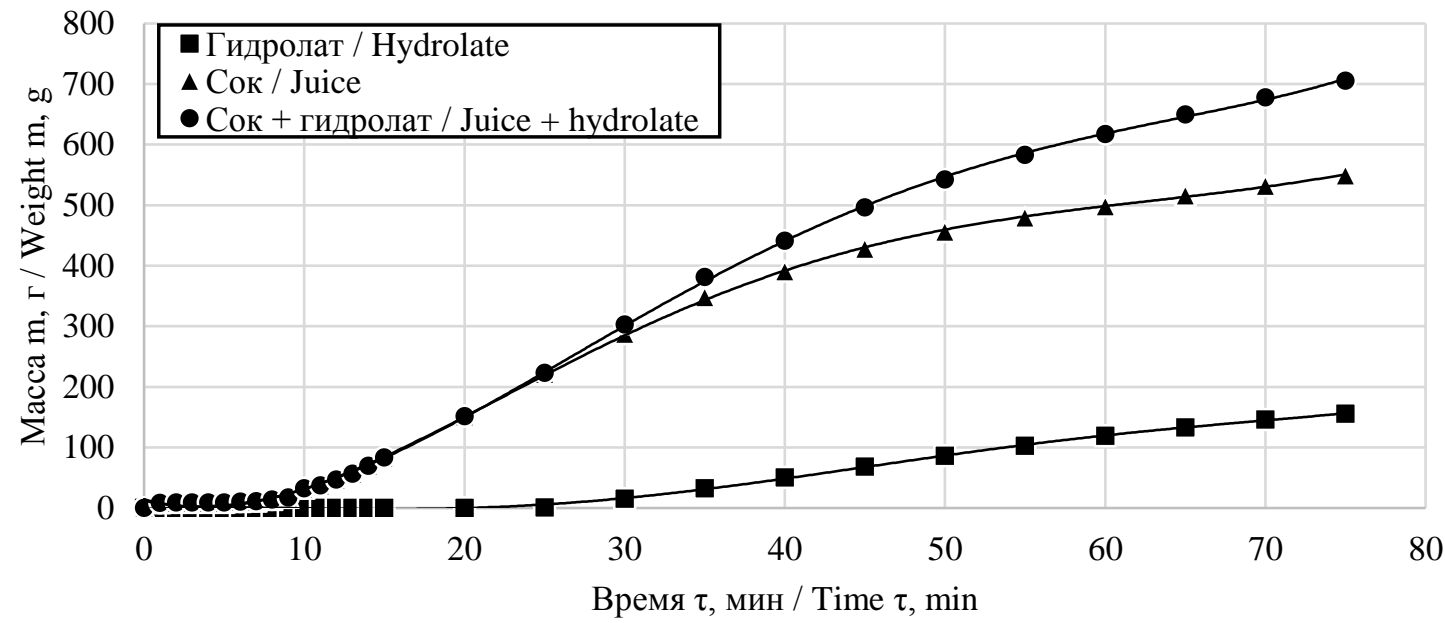

Рис. 3. Кинетика обезвоживания сырья с твердой фазой. ${ }^{3}$

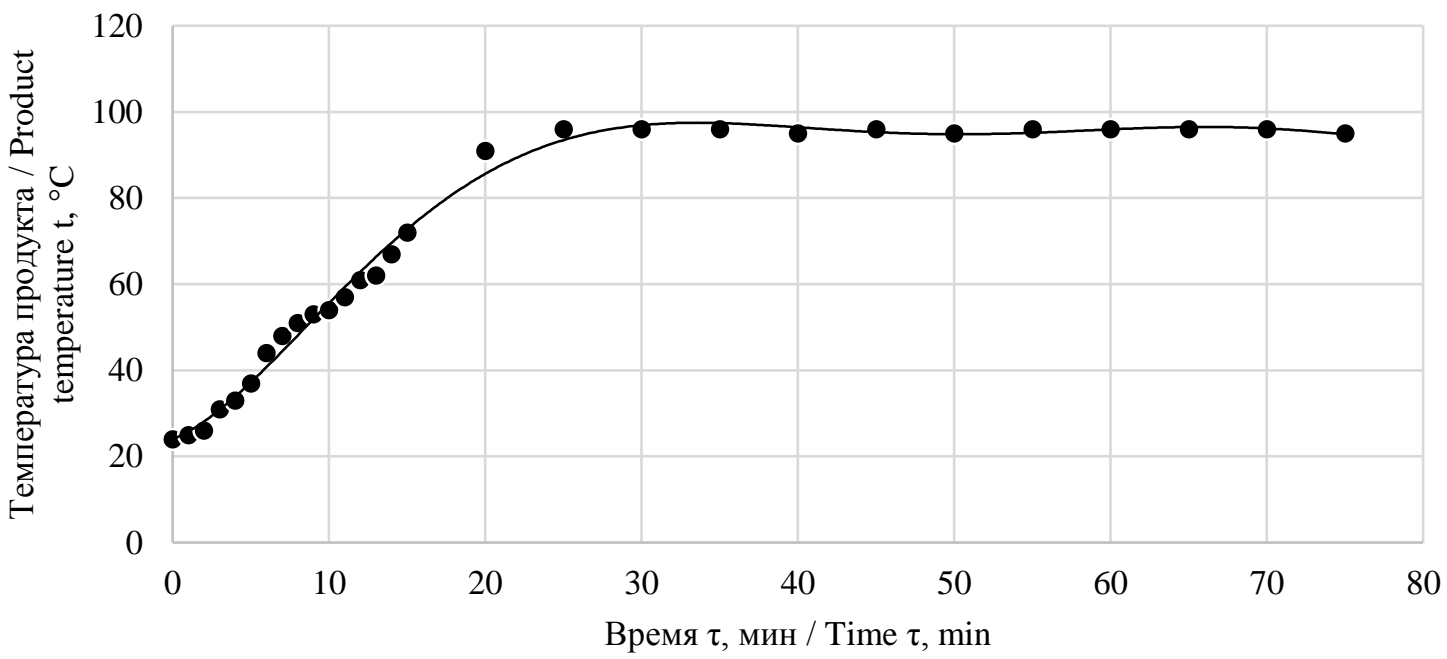

Рис. 4. Термограмма процесса обезвоживания. ${ }^{4}$ 
Опыты (рис. 3) проводились при атмосферном давлении в камере, температуры изменялись от начальной до $96^{\circ} \mathrm{C}$ (рис. 4). Сравнение результатов опытов показывает, что сок начал выходить из твердой фазы на 8 минуте (рис. 3), а температура поверхности в это время равнялась $50^{\circ} \mathrm{C}$ (рис. 4).

Это свидетельствует о том, что температура парообразования достигалась в глубине твердой фазы раньше, чем на поверхности.

Результаты исследований, проведенных на арбузе, (рис. 3) подтверждают сформулированную гипотезу. За время эксперимента выход сока оказался в 4,3 раза выше, чем пара (гидролата). Это свидетельствует о существенном снижении энергетических затрат в предложенном методе обезвоживания.

Таким образом, работа аппарата в режиме пародинамического эффекта имеет следующие достоинства: в 4 раза

уменьшаются затраты энергии на процесс дегидратации и сохраняется структура сырья. Самостоятельный интерес имеют задачи использования пародинамического эффекта, процесса фильтрационной дегидратации при переработке отходов пищевых производств.

\section{ИННОВАЦИОННЫЕ ЭНЕРГОТЕХНОЛОГИИ ЭКСТРАГИРОВАНИЯ И ДЕГИДРАТАЦИИ ПРИ ПЕРЕРАБОТКЕ ОТХОДОВ ПИЩЕВЫХ ПРОИЗВОДСТВ}

В качестве объектов исследований выбраны маслосодержащие отходы пищеконцентратных и масложировых производств. Это оболочки зерен кофе и кофейный шлам, жмых после производства масла амаранта, отработавшие реагенты (перлит, глина) в производстве масла подсолнечника. Схема переработки таких отходов на базе разработок лаборатории одинаковая (рис. 5).

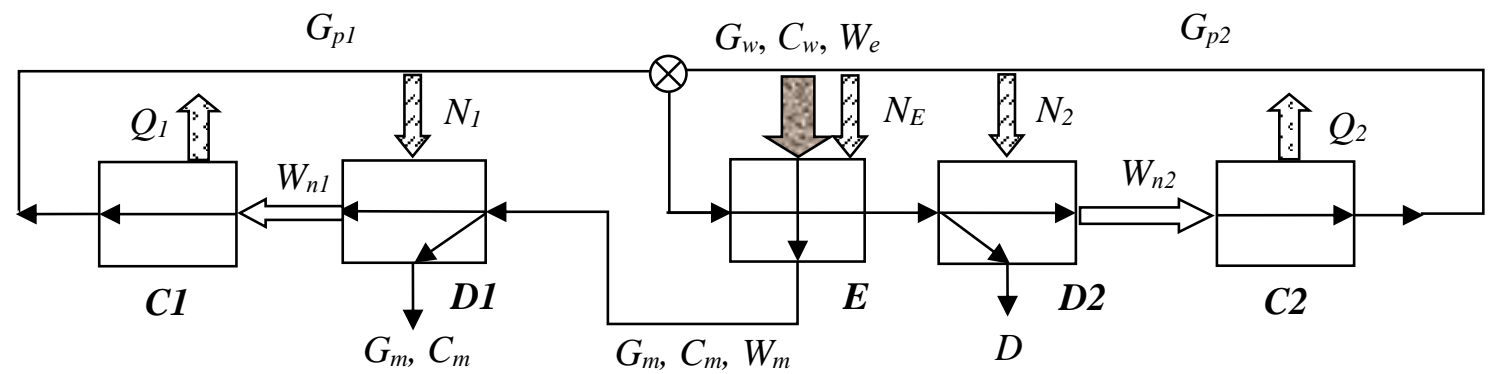

Рис. 5. Схема материальных и энергетических потоков. ${ }^{5}$

Задача технологической линии извлечь масло из отходов производства. Из маслосодержащего сырья, расход которого $G_{w}$, в экстракторе $E$ растворителем извлекается масло. Обезжиренный шлам (шрот) поступает в микроволновой дегидратор ( $D 1)$, где из него удаляются пары экстрагента. В дистилляторе $C l$ экстрагент конденсируется и возвращается в экстрактор $E$ потоком $G_{p 1}$. Аналогично из экстракта в дегидраторе ( D2) удаляется пар экстрагента и конденсируется в $C 2$. Раствор экстрагента $\left(G_{p 2}\right)$ подается в экстрактор $E$. Характеристика потоков приведена в табл. 1.

Характеристика материальных и энергетических потоков ${ }^{7}$

Таблица $1^{6}$.

\begin{tabular}{|c|c|l|l|}
\hline \multirow{2}{*}{ № } & $\begin{array}{l}\text { Символы } \\
\text { Symbols }\end{array}$ & \multicolumn{1}{|c|}{ Материальный / Material } & \multicolumn{1}{|c|}{ Энергетический / Energetic } \\
\cline { 3 - 4 } & $G_{w}$ & $\begin{array}{l}\text { Обезвоженные маслосодержащие } \\
\text { oтходы. / } \\
\text { Dehydrated oil-containing waste. }\end{array}$ & $\begin{array}{l}\text { Подводится энергия } N_{E}, \text { необходимая } \\
\text { для экстрагирования. / } \\
\text { The energy } N_{E} \text { required for the extraction is } \\
\text { supplied. }\end{array}$ \\
\hline 2 & $D$ & $\begin{array}{l}\text { Готовый продукт - масло, извлекается из } \\
\text { экстракта. / } \\
\text { Finished product - oil, removed from the } \\
\text { extract. }\end{array}$ & $\begin{array}{l}\text { Подводится энергия } N_{2}, \text { необходимая для } \\
\text { удаления растворителя. / } \\
\text { The energy } N_{2} \text { required for extractant } \\
\text { removing is supplied. }\end{array}$ \\
\hline
\end{tabular}


Продолжение таблицы $1^{8}$.

\begin{tabular}{|c|c|c|c|}
\hline \multirow{2}{*}{ № } & \multirow{2}{*}{$\begin{array}{l}\text { Символы } \\
\text { Symbols }\end{array}$} & \multicolumn{2}{|c|}{ Поток / Flow } \\
\hline & & Материальный / Material & Энергетический / Energetic \\
\hline 3 & $G_{m}, C_{m}, W_{m}$ & $\begin{array}{l}\text { Обезжиренный шлам, содержащий сухие } \\
\text { вещества }\left(C_{m}\right) \text { и экстрагент }\left(W_{m}\right) / \\
\text { Oil-free meal with solid matter }\left(C_{m}\right) \text { and } \\
\text { extractant }\left(W_{m}\right)\end{array}$ & \\
\hline 4 & $G_{m}, C_{m}$ & $\begin{array}{l}\text { Готовый продукт - сухой обезжиренный } \\
\text { шлам. / } \\
\text { Finished product - dry oil-free meal. }\end{array}$ & $\begin{array}{l}\text { Подводится энергия } N_{l} \text {, необходимая для } \\
\text { удаления растворителя. / } \\
\text { The energy } N_{l} \text { required for extractant } \\
\text { removing is supplied. }\end{array}$ \\
\hline 5 & $W_{n 1}$ & $\begin{array}{l}\text { Поток пара экстрагента из } \\
\text { дегидратора } D 1 . / \\
\text { Extractant vapor flow from the } \\
\text { dehydrator } D 1 \text {. }\end{array}$ & $\begin{array}{l}\text { Отводится энергия } Q_{1}, \text { необходимая для } \\
\text { конденсации паров в } C l . / \\
\text { The energy } Q_{1} \text { required for vapor } \\
\text { condensing to } C l \text { is rejected. }\end{array}$ \\
\hline 6 & $W_{n 2}$ & $\begin{array}{l}\text { Поток пара экстрагента из } \\
\text { дегидратора } D 2 . / \\
\text { Extractant vapor flow from the } \\
\text { dehydrator } D 2 \text {. }\end{array}$ & $\begin{array}{l}\text { Отводится энергия } Q_{2}, \text { необходимая для } \\
\text { конденсации паров в } C 2 . / \\
\text { The energy } Q_{2} \text { required for vapor } \\
\text { condensing to } C 2 \text { is rejected. }\end{array}$ \\
\hline 7 & $G_{p 1}, G_{p 2}$ & $\begin{array}{l}\text { Потоки раствора экстрагента. / } \\
\text { Liquid extractant flows. }\end{array}$ & \\
\hline
\end{tabular}

Перечень исходных образцов и результаты экспериментов приведены в табл. 2. В опытах в качестве экстрагента использовался этанол, сохранялись одинаковыми режимные параметры: мощность магнетрона - $425 \mathrm{BT}$, гидромодуль - 1:9, начальная масса продукта - 0,1 кг, температура процесса экстрагирования $-70-75^{\circ} \mathrm{C}$ и рабочее давление - 0,1 МПа.

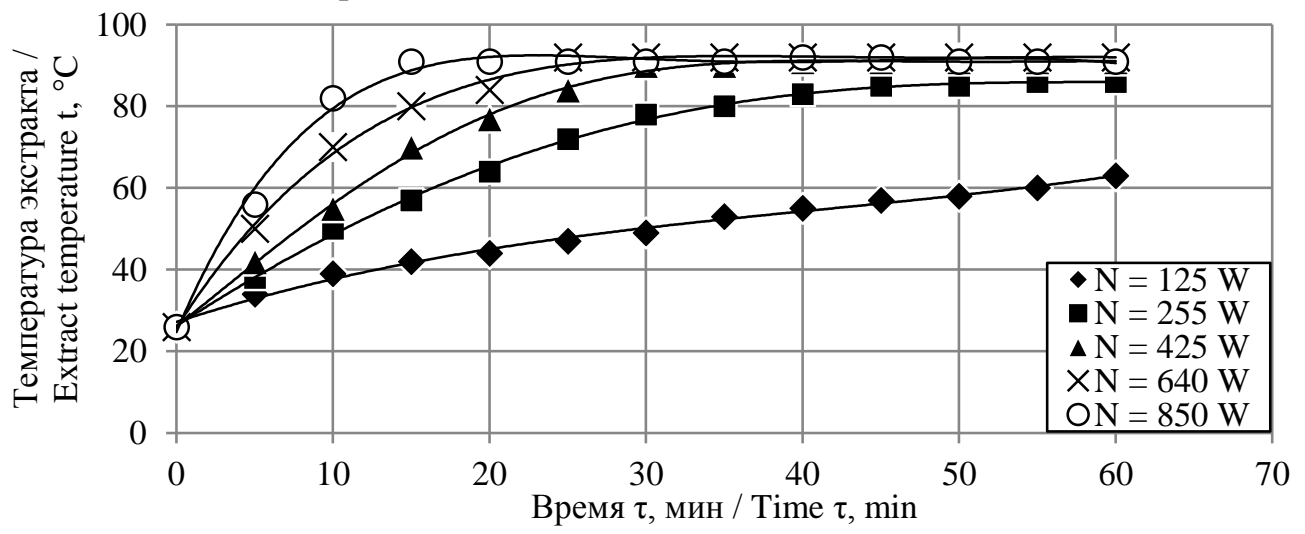

Рис. 6. Изменение температуры экстракта шелухи кофейных зерен во времени в зависимости от уровня подводимой мощности. ${ }^{9}$

Таблица $2^{10}$.

Характеристика объектов исследований ${ }^{11}$

\begin{tabular}{|c|c|c|}
\hline $\begin{array}{c}\text { Тип продукта } \\
\text { Product type }\end{array}$ & $\begin{array}{c}\text { Концентрация масла / } \\
\text { Oil concentration X, \% }\end{array}$ & $\begin{array}{c}\text { Macca мaсла m, г / } \\
\text { Oil mass m, g }\end{array}$ \\
\hline Шелуха кофейных зерен / Coffee beans husk & 0,8 & 5,2 \\
\hline Шрот из зерен амаранта / Amaranth-seed meal & 1,14 & 9,7 \\
\hline Перлит вымороженный / Perlite winterized & 1,35 & 10,3 \\
\hline Перлит не вымороженный / Perlite nonwinterized & 1,8 & 12,4 \\
\hline Глина с экзотическими маслами / Clay with exotic oils & 1,42 & 11,6 \\
\hline Глина с подсолнечным маслом / Clay with sunflower- \\
seed oil
\end{tabular}




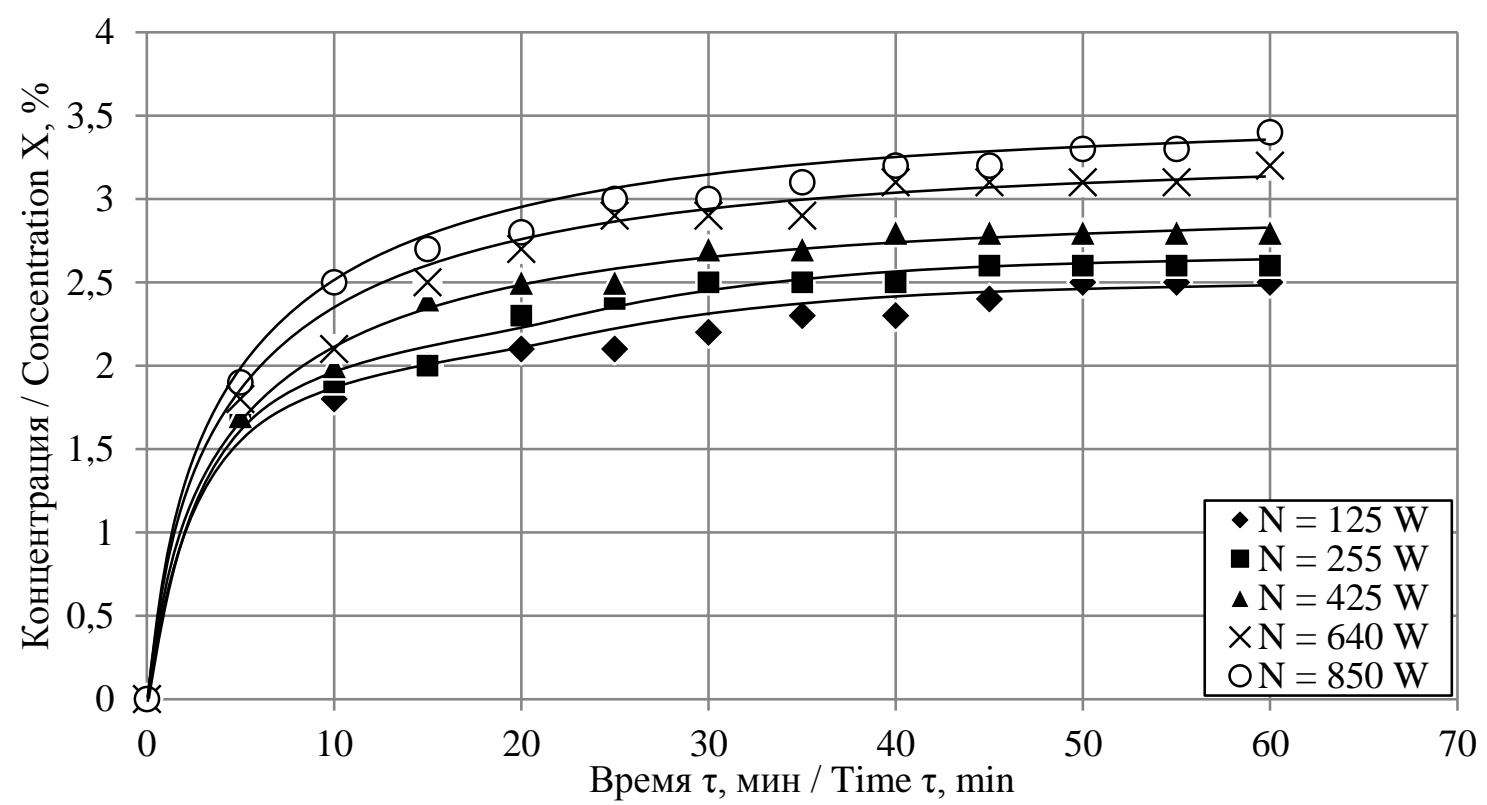

Рис. 7. Изменение концентраций экстракта шелухи кофейных зерен во времени в зависимости от уровня подводимой мощности. ${ }^{12}$

Результаты экспериментов показали (рис. 6, 7), что с увеличением уровня подводимой мощности МВ энергии, как следствие, растет температура продукта, что способствует интенсификации процесса экстрагирования. Концентрации полученных экстрактов (табл. 2) свидетельствуют о значительном содержании масла в отходах, что подтверждает целесообразность выбора вышеуказанных образцов. Кинетика процесса экстрагирования отображена B зависимости на рис. 8. Видно, что основная доля экстрактивных веществ переходит в раствор в течение 30 минут и в дальнейшем наблюдается медленный прирост концентрации. Таким образом, целесообразно сократить время экстрагирования вдвое с целью минимизации энергозатрат, сохранив при этом возможность достижения высокой конечной концентрации.

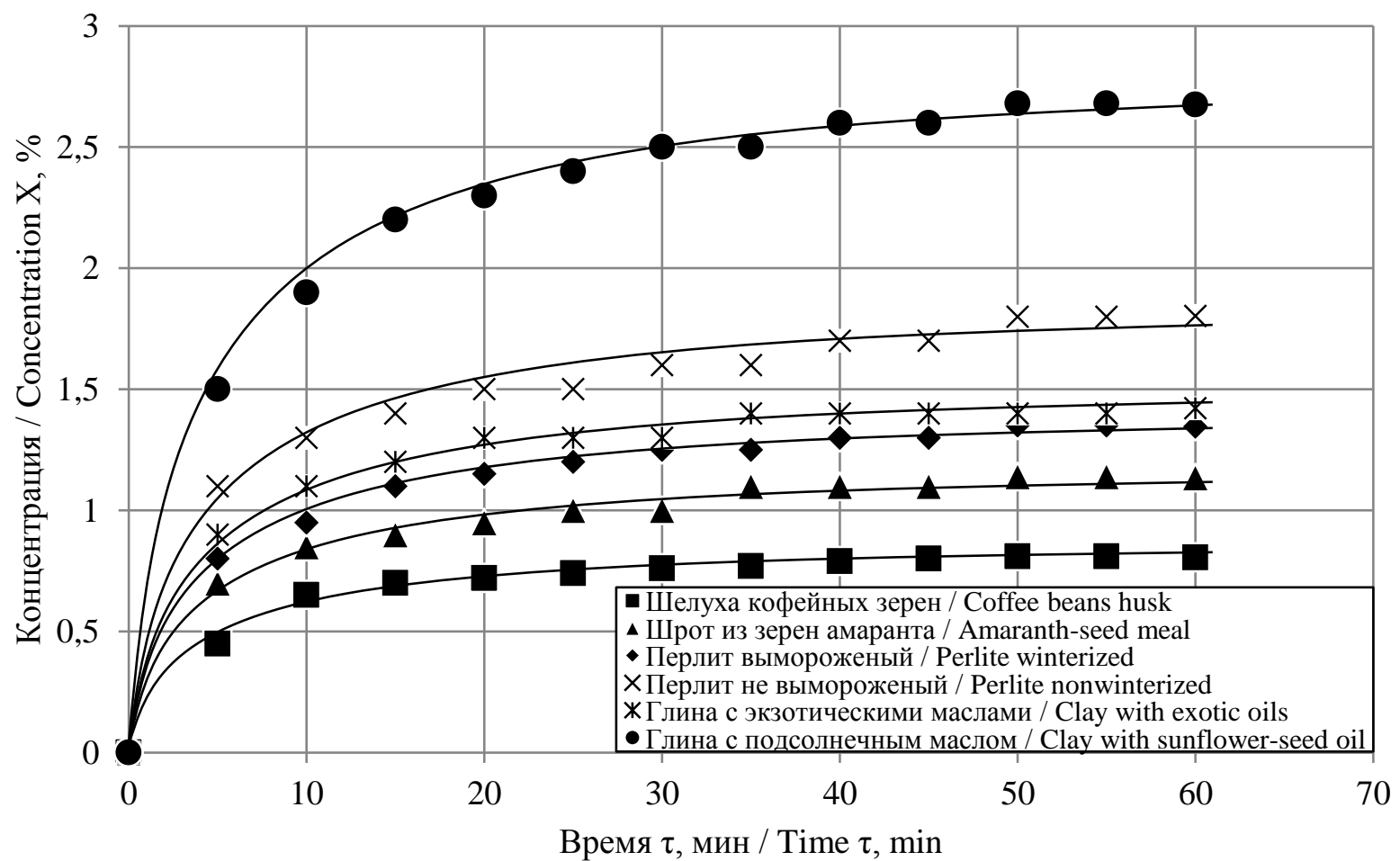

Рис. 8. Изменение концентраций во времени в зависимости от типа продукта. ${ }^{13}$ 
Таблица $3^{14}$.

Результаты проведения анализа полученных образцов 15

\begin{tabular}{|c|l|c|c|c|c|}
\hline № & \multicolumn{1}{|c|}{ Name of acid } & $\begin{array}{c}\text { Statistic DW } \\
\text { by SFO }\end{array}$ & $\begin{array}{c}\text { Standart, } \\
\% \text { RSFO }\end{array}$ & $\begin{array}{c}\text { Oil from perlite } \\
\text { winterized }\end{array}$ & $\begin{array}{c}\text { Oil from perlite } \\
\text { nonwinterized }\end{array}$ \\
\hline 1 & Myristic Acid Methyl Ester (C14:0) & up to 0,2 & up to 0,2 & 0,1 & 0,1 \\
\hline 2 & Palmitic Acid Methyl Ester (C16:0) & $6,2-7,4$ & $5,0-7,6$ & 7,2 & 6,8 \\
\hline 3 & Palmitoleic Acid Methyl Ester (C16:1) & up to 0,3 & up to 0,3 & 0,1 & 0,1 \\
\hline 4 & Stearic Acid Methyl Ester (C18:0) & $3,5-3,9$ & $2,7-6,5$ & 2,9 & 3,5 \\
\hline 5 & Oleic Acid Methyl Ester (C18:1n9c) & $23,0-26,7$ & $14,0-39,4$ & 0,1 & 22,6 \\
\hline 6 & Linoleic Acid Methyl Ester (C18:2n6c) & $61,3-64,7$ & $48,3-74,0$ & 20,5 & 65,7 \\
\hline 7 & Arachidic Acid Methyl Ester (C20:0) & $0,1-0,5$ & $0,1-0,5$ & 0,2 & 0,2 \\
\hline 8 & cis-11-Eicosenoic Acid Methyl Ester (C20:1) & up to 0,3 & up to 03 & 0,1 & 0,1 \\
\hline 9 & Linolenic Acid Methyl Ester (C18:3n3) & up to 0,3 & up to 0,3 & 0,5 & 0,1 \\
\hline
\end{tabular}

Результаты анализа (табл. 3) свидетельствуют, что представленные в работе инновационные технологии переработки отходов пищевых производств обеспечивают экологическую безопасность конечного продукта и его соответствие нормам качества.

\section{ВЫВОДЫ}

Современный рынок пищевых продуктов характеризуется стабильным ростом интереса к концентратам. Жидкие концентрированные продукты выпускаются на аппаратах вакуумвыпарного принципа, а сухие и твердые преимущественно на сушилках конвективного типа. Вместе с тем, традиционное выпарное и сушильное оборудование требует развития. Многоступенчатые вакуум-выпарные аппараты

удовлетворительными характеризуются энергетическими характеристиками, но ограничены по параметрам концентрации готового продукта. Физический принцип их работы теплопередача - не позволяет им работать интенсивно в условиях повышенных концентраций. Физический принцип работы сушильного оборудования - диффузия требует повышенных затрат энергии. Авторы выдвинули гипотезу и доказали, что переход от условий теплопередачи к условиям объемного подвода энергии (т.е. переход к источникам энергии с граничными условиями 2 рода) позволит существенно расширить возможности инновационной выпарной техники:
- получать в условиях действия электромагнитных полей концентрацию готового продукта на уровне $92 \ldots 95^{\circ}$ brix;

- работать с сырьем, содержащим твердую фазу и организовать из твердой фазы влажного сырья, кроме традиционного потока пара, специфический поток жидкости. Этот поток жидкости в 4,3 раза превышал массовый расход пара, т.е. жидкая фаза отводилась механически и не требовала расхода энергии на фазовый переход.

Вторым важным результатом работы является доказательство эффективности использования электромагнитных источников энергии для интенсификации процесса экстрагирования при утилизации отходов пищевых производств. Удовлетворительные результаты получены как при экстрагировании целевых компонентов из отходов пищеконцентратного производства (кофейной шелухи, шлама), при утилизации жмыха амаранта, так и при регенерации перлита и глины в технологиях подсолнечного масла.

Разработанная инновационная техника экстрагирования и дегидратации имеет широкие межотраслевые перспективы.

\section{APPENDIX 1 (ПРИЛОЖЕНИЕ 1)}

${ }^{1}$ Fig. 1. Scheme of dehydration process. ( $a$ - physical model of plant; $b$ - model of "vapor and dynamic effect").

${ }^{2}$ Fig. 2. Scheme of experimental stand. ( $V P$ - vacuum pump, $M-$ manometer, $V$ - vapor, $C$ - condencer, $C M$ - cooling machine, $E G$ - electromagnetic generator, $T P$ - teflon plate, $E S$ - electronic scale, $J C-$ juice collector, $C C-$ condensate collector, $M C C-$ 
measuring and calculating complex, $S 1, S 2$ - weight sensors, $S 3, S 4$ - temperature sensors).

${ }^{3}$ Fig. 3. Kinetic of raw material with solid phase dehydration.

${ }^{4} \mathbf{F i g}$. 4. Thermogram of dehydration process.

${ }^{5}$ Fig. 5. Scheme of material and energy flows. ( $E-$ extractor, D1, D2-dehydrator, $C 1, C 2$ - condenser, $G_{r}$ - raw material mass, $C_{r}$ - initial oil concentration in raw material, $W_{e}$ - extractant mass, $G_{m}$ - meal mass, $C_{m}$ - final oil concentration in meal, $W_{m}$ - extract mass in meal, $W_{n 1}, W_{n 2}$ - extractant vapor mass after dehydrator, $G_{p 1}, G_{p 2}-$ extractant mass after condenser, $D$ - oil mass, $N_{E}$ - energy supply for exraction, $N_{l}, N_{2}$ - energy supply for extractant evaporation, $Q_{1}, Q_{2}-$ energy rejection for extractant vapor condensation).

${ }^{6,7}$ Table 1. Characteristic of material and energy flows. ${ }^{8}$ Table 1 (Continued).

${ }^{9}$ Fig. 6. Change of temperature of coffee beans husk extract in time in dependence of energy supply level. ${ }^{10,11}$ Table 2. Characteristic of researching objects.

${ }^{12}$ Fig. 7. Change of concentration of coffee beans husk extract in time in dependence of energy supply level.

${ }^{13}$ Fig. 8. Change of concentrations in time in dependence of product type.

${ }^{14,15}$ Table 3. Results of analysis of obtained samples.

\section{Литература (References)}

[1] Goodman E.M., Redmond J., Elia D., Harris S.R., Augustine M.B., Hand R.K. Practice Roles and Characteristics of Integrative and Functional Nutrition Registered Dietitian Nutritionists. Journal of the Academy of Nutrition and Dietetics, 2018, vol. 118, no. 12, pp. 2356-2369. doi: 10.1016/j.jand.2018.03.027

doi: 10.5281/zenodo.3970438

[2] Gryshova I., Nikoliuk O., Shestakovska T. The Organic Production In The Context Of Improving The Ecological Safety Of Production Of The Food Industry. Journal of Food Science And Technology-Ukraine, 2017, vol. 11, no. 4, pp. 103112. doi: 10.15673/fst.v1li4736

[3] Bazhenov A.A., Mizikovsky, I.E., Garina, E.P., Kuznetsov, V.P., Gavrilov, A.I. Normal Flow of Resources as a Basis for Improving the Quality of Final Financial Information. Conference on Future of the Global Financial System - Downfall or Harmony, April 13-14 2018, vol. 57, pp. 309315. doi: 10.1007/978-3-030-00102-5_32

[4] Trojahn S. Logistics Strategies For Resource Supply Chains. Transport And Telecommunication Journal, 2018, vol. 19, no. 3, pp. 244-252. doi: $10.2478 / \mathrm{ttj}-2018-0021$

[5] Landgrebe D., Kräusel V., Bergmann M., Werner, M., Rautenstrauch A. Conserving Resources in Production - Breaking New Ground. Procedia
Manufacturing, 2017, vol. 8, pp. 619-626. doi: 10.1016/j.promfg.2017.02.079

[6] Irmak S. Biomass as Raw Material for Production of High- Value Products. Biomass Volume Estimation and Valorization for Energy, 2017, pp. 201-225. doi:10.5772/65507

[7] Elik A., Yanik D.K., Mascan M., Göğüş F. Influence of three different concentration techniques on evaporation rate, color and phenolics content of blueberry juice. J Food Sci Technol, 2016, vol. 53, no. 5, pp. 2389-2395.

doi: 10.1007/s13197-016-2213-0

[8] Bozkir H., Baysal T. Concentration of apple juice using a vacuum microwave evaporator as a novel technique: Determination of quality characteristics. J Food Process Eng, 2017, pp. 19. doi: 10.1111/jfpe.12535

[9] Bozkir H., Baysal T. Concentration of orange juice by vacuum microwave evaporator. $3 \mathrm{rd}$ International Congress on Food Technology; October 10-12, 2018 Nevşehir/Turkey. - P. 426.

[10]Dinçer C., Çam I.B., Torun M., Gulmez H.B., Topuz A. Mathematical modeling of concentrations of grape, pomegranate and black carrot juices by various methods. The Journal of Food, 2019, vol. 44, no. 6, pp. 1092-1105.

doi: 10.15237/gida.GD19080

[11] Alvi T., Khan M.K.I., Maan A.A., Nazir A., Ahmad M.H., Khan M.I., Qureshi S. Modelling and Kinetic Study of Novel and Sustainable Microwave-Assisted Dehydration of Sugarcane Juice. Processes, vol. 10, no. 7, 712. doi: 10.3390/pr7100712

[12] Asghar M., Yusof Y., Mokhtar M. et al. Processing of coconut sap into sugar syrup using rotary evaporation, microwave, and open-heat evaporation techniques. J Sci Food Agric, 2020, pp. 4012-4019. doi: 10.1002/jsfa.10446

[13]Lohrasbi-Nejab S., Shahedi M., Fathi M. Comparative study of microwave-vacuum, microwave and convectional evaporation methods on physicochemical properties of barberry juice. $J$ Agr Sci Tech, 2021, vol. 23, no. 2, pp. 307-317.

[14] Naderi B., Maghsoudlou Y., Aminifar M. et al. Investigation on the changes in color parameters and turbidity of cornelian cherry (cornus mass L) produced by microwave and convectional heating. Nutrition and Food Sciences Research, 2015, vol. 4, no. 2, pp. 39-46.

[15] Yousefi S., Emam-Djomeh Z., Mousavi A. Askari G.R.. Comparing the effects of microwave and conventional heating methods on the evaporation rate and quality attributes of pomegranate (Punica granatum L.) juice concentrate. Food Bioprocess Technol, 2011, vol. 5, pp. 1328-1339. doi: 10.1007/s11947-0110603-X 
[16] Fazaeli M., Hojjatpanah G., Emam-Djomeh Z. Effects of heating method and conditions on the evaporation rate and quality attributes of black mulberry (Morus nigra) juice concentrate. J Food Sci Technol, 2011, vol. 50, no. 1, pp. 35-43. doi: 10.1007/s13197-011-0246-y

[17] Qi C.Tianyang G., Jian Y., Renyi L., Peng W., Qiong W., Shaotong J., Yiyang D. MicrowaveAssisted Extraction Combined with Ultra-HighPerformance Liquid Chromatography and Quadrupole/Q-Exactive High-Resolution Mass Spectrometry for the Determination of Main Flavor Substances in Green Tea. Journal of AOAC International, 2020, vol. 103, no. 2, pp. 428-432. doi:10.5740/jaoacint.19-0265

\section{Сведения об авторах.}
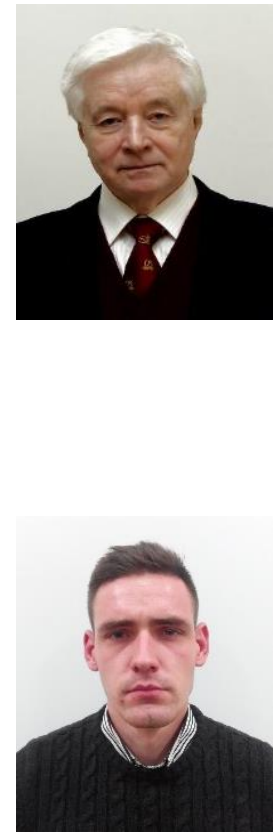

\section{Щербич Максим}

Владимирович, аспирант.

Сфера научных интересов: экстрагирование, энергоэффективность.

г. Одесса, Украина

E-mail:

poem.onaft@gmail.com

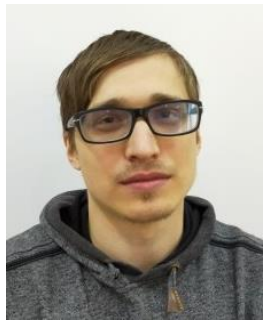

[18] Taşkin O., Izli N. Effect of Microwave, Infrared and Freeze Drying Methods on Drying Kinetics, Effective Moisture Diffusivity and Color Properties of Turmeric. Journal of Agricultural Sciences, 2019, no. 25, pp. 334-345. doi: 10.15832/ankutbd.439434

[19] Ozturk S., Şakıyan O., Alifaki Y.O. electric properties and microwave and infraredmicrowave combination drying characteristics of banana and kiwifruit. J. Food Process Eng., 2016. doi: 10.1111/jfpe.12502

[20]Planovskii A.N. Nikolaev P.I. Protsessy I apparaty himicheskoi $i$ neftehimicheskoi tehnologii. 3rd ed. Moscow, Himiya, 1987. 495 p.

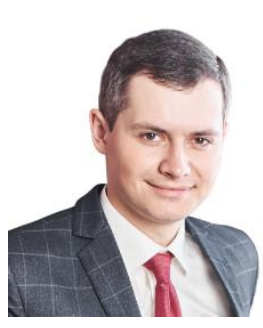

Сиротюк

Илья

Вадимович, аспирант. Сфера научных интересов: процессы тепломассообмена, экстрагирование, обезвоживание пищевых продуктов, энергоэффективность г. Одесса, Украина

E-mail:

ilyxin09@gmail.com

Акимов Александр
Викторович, аспирант.
Сфера научных
интересов:
экстрагирование,
выпарка, микроволновые
технологии
г. Одесса, Украина
E-mail:
akimch27@gmail.com

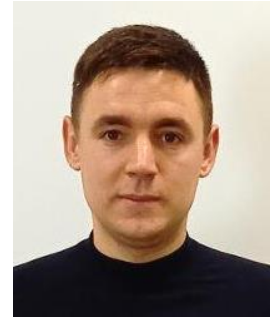

Поян Александр
Сергеевич,
КИПиА ООО «Дельта
Вилмар Украина». Сфера
научных интересов: пере-
работка отходов пищевых
производст. г. Южный,
Украина.
Е-mail:
Poyan.aleksandr@gmail.com

